\title{
Performance of Whole-Body PET/CT for the Detection of Distant Malignancies in Various Cancers: A Systematic Review and Meta-Analysis
}

\author{
Guozeng $\mathrm{Xu}^{* 1-3}$, Lin Zhao*1,3,4, and Zhiyi $\mathrm{He}^{1,3}$ \\ ${ }^{I}$ Evidence-Based Medical Center, The First Affiliated Hospital of Guangxi Medical University, Nanning, People's Republic of China; \\ ${ }^{2}$ Department of Radiation Oncology, Guangxi Zhuang Autonomous Region Tumor Hospital, Nanning, People's Republic of China; \\ ${ }^{3}$ Department of Respiratory Medicine, The First Affiliated Hospital of Guangxi Medical University, Nanning, People's Republic of \\ China; and ${ }^{4}$ Graduate School of Guangxi Medical University, Nanning, People's Republic of China
}

\begin{abstract}
At present, there is no ideal imaging modality for the diagnosis of distant metastases and second primary cancers in cancer patients. We aimed to assess the accuracy of whole-body $\mathrm{PET} / \mathrm{CT}$ for the overall assessment of distant malignancies in patients with various cancers. Methods: Studies about wholebody PET/CT for the detection of distant malignancies in cancer patients were systematically searched in MEDLINE and EMBASE. We determined sensitivities and specificities across studies, calculated positive and negative likelihood ratios, and constructed summary receiver operating characteristic curves using hierarchical regression models for whole-body PET/CT. Results: Across 41 studies (4,305 patients), the sensitivity and specificity of whole-body PET/CT were $0.93(95 \%$ confidence interval [Cl], 0.88-0.96) and $0.96(95 \% \mathrm{Cl}, 0.95-$ 0.96), respectively. Subgroup analysis showed that the sensitivity and specificity of whole-body PET/CT for various cancers, respectively, were as follows: head and neck cancer, 0.90 (95\% $\mathrm{Cl}, 0.83-0.95)$ and $0.95(95 \% \mathrm{Cl}, 0.94-0.96)$; lung cancer, 0.91 (95\% Cl, 0.76-0.97) and 0.96 (95\% Cl, 0.94-0.98); breast cancer, 0.97 (95\% Cl, 0.93-0.99) and 0.95 (95\% Cl, 0.90-0.97); and cancer of digestive system, $0.92(95 \% \mathrm{Cl}, 0.68-0.98)$ and 0.97 (95\% Cl, 0.91-0.99). Conclusion: Whole-body PET/CT has excellent diagnostic performance for the overall assessment of distant malignancies in patients with various cancers, especially head and neck cancer, breast cancer, and lung cancer.
\end{abstract}

Key Words: whole-body PET-CT; distant malignancies; cancers; meta-analysis

J Nucl Med 2012; 53:1847-1854

DOI: 10.2967/jnumed.112.105049

$\mathbf{T}$ he presence of distant metastases is one of the most important prognostic factors in most cancer patients. Most tumors are classified according to the TNM staging system, and treat-

\footnotetext{
Received Feb. 27, 2012; revision accepted Jul. 2, 2012.

For correspondence or reprints contact: Zhiyi He, Department of Respiratory Medicine, The First Affiliated Hospital of Guangxi Medical University, Nanning 530021, People's Republic of China.

E-mail: lichunyan910910@sina.com

${ }^{*}$ Contributed equally to this work.

Published online Oct. 16, 2012.

COPYRIGHT @ 2012 by the Society of Nuclear Medicine and Molecular Imaging, Inc.
}

ment is modified when distant metastases are present. Disease localized to primary sites and to regional lymph nodes is generally treated with curative strategies, including surgery, chemotherapy, and radiotherapy. In contrast, palliative treatment of patients with metastatic disease consists of less aggressive strategies. Moreover, distant metastases usually occur late during the course of cancer, whereas second primary cancers may be found even in early-stage patients. Early detection of distant metastases and second primary cancers is a fundamental precondition for guiding precise staging and optimal management.

Conventional imaging procedures (such as chest radiography, CT, abdominal ultrasonography, and bone scan) are commonly used to detect distant metastases and second primary cancers in patients with various cancers $(1,2)$. However, conventional imaging procedures often do not reliably characterize the extent of disease because it is difficult to identify small distant lesions on the basis of morphologic criteria and to distinguish potential metastatic lesions from benign findings. ${ }^{18} \mathrm{~F}-\mathrm{FDG}$ PET is a functional imaging modality that is based on the increased glucose metabolism of malignant cells. However, anatomic information concerning distant lesions is limited on ${ }^{18} \mathrm{~F}$-FDG PET images, and the resolution is insufficient to detect small lesions. The introduction of PET/CT scanners combined the functional data of PET with the detailed anatomic information of CT into a single examination. In several previous studies, ${ }^{18} \mathrm{~F}-\mathrm{FDG}$ PET/CT was shown to be more sensitive and specific than conventional imaging procedures for the detection of distant malignancies in cancer patients at initial staging before treatment or restaging after treatment (1-5). Although many studies about wholebody PET/CT for various cancers were done, the results were still controversial and inconclusive. Here, we undertook a meta-analysis to evaluate the diagnostic performance of whole-body PET/CT for detecting distant malignancies in patients with various cancers.

\section{MATERIALS AND METHODS}

\section{Search Strategy}

We searched for studies evaluating whole-body PET/CT for the overall assessment of distant metastases with or without second 
primary cancers in patients with various cancers. Articles were identified with a search of MEDLINE and EMBASE from January 1, 2000, to April 30, 2012. We used a search algorithm that was based on a combination of text words: (CT OR "computed tomography") AND (PET OR "positron emission tomography") AND (neoplasm OR cancer OR carcinoma) AND (staging OR "distant metastases"). We had no language restrictions for searching relevant studies. References in the retrieved articles were also screened for additional studies. Authors of eligible studies were contacted and asked to supplement additional data when key information relevant to the meta-analysis was missing.

\section{Study Selection}

We considered studies using ${ }^{18} \mathrm{~F}-\mathrm{FDG}$ PET/CT for the overall assessment of distant malignancies in cancer patients. Inclusion criteria were as follows: ${ }^{18} \mathrm{~F}$-FDG PET/CT was used as a diagnostic tool in cancer patients of all ages regardless of primary sites and treatment status; there were sufficient data to reconstruct a $2 \times 2$ table such that the cells in the table could be labeled as showing true-positive, false-positive, true-negative, and false-negative results; there was a minimal sample size of 10 cancer patients, including both patients with and patients without distant metastases; analysis was done at the patient level; studies with both retrospective and prospective designs were included in this metaanalysis; and histopathologic analysis or clinical and imaging follow-up was used as the gold standard to assess diagnostic performance. We excluded studies that focused exclusively on second primary cancers and studies from the same study group. We also excluded studies with verification bias, that is, those in which the reference standard was used only for subsets of patients based on positive PET/CT results.

\section{Data Extraction}

Two reviewers extracted data from eligible studies independently and resolved discrepancies by discussion. A third investigator settled any remaining discrepancies. For each report, we recorded the author names, year of publication, country of origin, number of eligible patients, type of eligible patients (those with primary cancer or recurrent cancer), study design (prospective or retrospective), type of cancer (head and neck, lung, breast, digestive system, urogenital system, melanoma, or others), and definition of positive PET/CT results (both qualitative and quantitative, qualitative, or unclear). For each study, we recorded the number of true-positive, false-positive, true-negative, and false-negative findings for whole-body PET/CT using histopathologic analysis or clinical and imaging follow-up as the reference standard.

\section{Quality Assessment}

To evaluate the quality, applicability, and reporting of the studies, we used a tool for the quality assessment of studies of diagnostic accuracy (QUADAS); this tool was recently proposed to assess the quality of studies of diagnostic accuracy included in a meta-analysis. The QUADAS tool included 14 items, each of which was assessed as "yes" or "no."

\section{Statistical Analysis}

We used bivariate regression models to obtain weighted overall estimates of sensitivity and specificity as the main outcome measures and to construct hierarchic summary receiver operating characteristic (HSROC) curves for whole-body PET/CT. On the basis of random-effects models, this bivariate approach accounted for potential between-study heterogeneity and incorporated the correlation between sensitivity and specificity. Overall sensitivity and specificity and their 95\% confidence intervals (CIs) were calculated on the basis of the binominal distributions of truepositive and true-negative findings. By using the pooled sensitivities and specificities, we also calculated positive likelihood ratios (PLRs) and negative likelihood ratios (NLRs) for whole-body PET/CT. Discriminating ability is better with higher PLRs and lower NLRs. Although there is no absolute cutoff, a good diagnostic test may have PLRs of greater than 10.0 and NLRs of less than 0.1 .

We used the summary estimates of sensitivity and specificity obtained in the meta-analysis for cancer patients to calculate negative predictive values (that is, the probability that a patient does not have distant malignancies when the test results are negative) for whole-body PET/CT when the prevalences of distant malignancies in the population were assumed to be $10 \%, 20 \%$, and $30 \%$.

We investigated the effect of heterogeneity on the diagnostic accuracy of whole-body PET/CT by subgroup analysis. Analysis of the covariates included the type of eligible patients (primary cancer vs. recurrent cancer), prevalence of distant malignancies (high prevalence $[\geq 15 \%]$ vs. low prevalence $[<15 \%]$ ), number of items assessed as "yes" in the QUADAS tool (high quality $[\geq 12]$ vs. low quality $[<12]$ ), imaging analysis (both quantitative and qualitative vs. qualitative), study design (prospective vs. retrospective), and primary site (head and neck, lung, breast, and digestive system). For studies that included both patients with primary cancer and patients with recurrent cancer, the relevant subsets of patients were included in the summary calculations for each subgroup when the data could be split into such subsets.

We also compared the performance of whole-body PET/CT with that of conventional imaging procedures using the same bivariate regression models. This analysis included all data regardless of the types of conventional imaging procedures compared.

All analyses were conducted with Stata version 11.0 (Stata Corp.).

\section{RESULTS \\ Eligible Studies}

The electronic search yielded 8,091 articles; 8,018 were excluded after reading of the abstract because they did not present any diagnostic information. We screened in full text 73 articles and rejected 32; thus, 41 articles (1-41) were eligible for the meta-analysis. Reasons for exclusion are shown in Figure 1. Of the 41 studies, 22 (53.7\%) were described as being prospective. All 41 studies (4,305 patients) were analyzed for the diagnostic accuracy of whole-body PET/CT for the detection of distant malignancies (Table 1). In 21 studies only patients with primary cancer were enrolled, in 14 studies only patients with recurrent cancer were enrolled, and in 6 studies mixed patient populations were enrolled. We were able to extract subgroup data (for primary cancer and recurrent cancer) from $2(6,20)$ of the studies with mixed populations. In 17 studies, whole-body PET/CT-positive results were stated to have been assessed in a qualitative manner, whereas in 19 studies, they were stated to have been assessed by both quantitative and qualitative manners. In 5 studies (33$35,38,41)$, the manner used for assessment was not stated. The prevalence of distant malignancies in the included studies ranged from $5.0 \%$ to $80.8 \%$. If $15 \%$ were used as a cutoff for high prevalence versus low prevalence, $41.5 \%$ of the 41 studies would have had a prevalence of less than $15 \%$. 


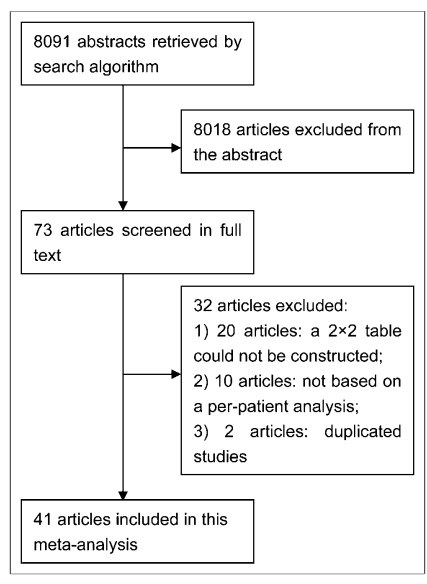

FIGURE 1. Flow chart of search for eligible studies.

\section{Quality Assessment}

We assessed the quality of the 41 articles according to the 14-item QUADAS tool. Eight of the 14 items could be scored for all included articles: clear selection criteria (item 2), acceptable reference standard (item 3), partial verification (item 5), incorporation bias (item 7), masking to reference test results (item 10), availability of clinical data that would be available in clinical practice when using the index test (item 12), reporting of uninterpretable results (item 13), and explanation of withdrawals from the study (item 14). No study $(0 \%)$ reported that all patients received the same reference test regardless of the index test result (item 6) or that the reference standard was masked to the index test result (item 11). Representative spectrum (item 1: was the spectrum of patients representative of the patients who will receive the test in practice?) was not present in 34.2\% $(2,4,9,16,18-21,26,28,29,34,38,39)$ of the 41 articles. Acceptable delay between tests (item 4 ) was not reported in $4.9 \%$ $(20,39)$ of the 41 articles. The execution of the index test in detail (item 8) was not present in $12.2 \%(33-35,38,41)$ of the 41 articles. The reference standard in detail (item 9) was not present in $4.9 \%(22,28)$ of the 41 articles. The number of items assessed as "yes" in the QUADAS tool would have been less than 12 in $43.9 \%$ of the 41 studies if 12 were used as a cutoff for high quality versus low quality,

\section{Diagnostic Accuracy of Whole-Body PET/CT}

When we considered all 41 studies (4,305 patients) with data on a per-patient basis $(1-41)$, the ${ }^{18} \mathrm{~F}-\mathrm{FDG}$ PET/CT sensitivity was 0.93 (95\% confidence interval [CI], 0.88$0.96)$ and the specificity was 0.96 (95\% CI, 0.95-0.96). The type of eligible patients, prevalence of distant malignancies, quality scoring, imaging analysis, and study design did not statistically significantly influence the reported sensitivities and specificities of ${ }^{18} \mathrm{~F}$-FDG PET/CT $(P>0.05)$ (Table 2).

Likelihood ratio syntheses yielded overall PLR of 20.8 (95\% CI, 16.8-25.8) and NLR of 0.08 (95\% CI, 0.05-0.13). Our data showed that the HSROC curve was positioned near the desirable upper left corner and that the overall weighted area under the curve was 0.97 (95\% CI, 0.95-0.98), indicating a high level of overall accuracy (Fig. 2).
When the prevalences of distant malignancies in cancer patients were assumed to be $10 \%, 20 \%$, and $30 \%$, the negative predictive values for ${ }^{18} \mathrm{~F}-\mathrm{FDG}$ PET/CT were 0.99 , 0.98 , and 0.97 , respectively.

\section{Diagnostic Accuracy of Whole-Body PET/CT for Various Cancers}

Head and Neck Cancer. When we considered all 16 studies (1,800 patients) with data on head and neck cancer (1,6-20), the pooled sensitivity, specificity, PLR, and NLR of ${ }^{18} \mathrm{~F}-\mathrm{FDG}$ PET/CT were 0.90 (95\% CI, 0.83-0.95), 0.95 (95\% CI, 0.94-0.96), 19.0 (95\% CI, 14.6-24.7), and 0.10 (95\% CI, 0.06-0.18), respectively.

Lung Cancer. When we considered all 5 studies (578 patients) with data on lung cancer (22-26), the pooled sensitivity, specificity, PLR, and NLR of ${ }^{18} \mathrm{~F}-\mathrm{FDG}$ PET/CT were 0.91 (95\% CI, 0.76-0.97), 0.96 (95\% CI, 0.94-0.98), 25.9 (95\% CI, 15.4-43.6), and 0.09 (95\% CI, 0.03-0.26), respectively.

Breast Cancer. When we considered all 5 studies (547 patients) with data on breast cancer $(2,28-31)$, the pooled sensitivity, specificity, PLR, and NLR of ${ }^{18} \mathrm{~F}-\mathrm{FDG}$ PET/CT were 0.97 (95\% CI, 0.93-0.99), 0.95 (95\% CI, 0.90-0.97), 18.5 (95\% CI, 10.0-34.1), and 0.03 (95\% CI, 0.01-0.07), respectively.

Cancers of Digestive System. When we considered all 6 studies (379 patients) with data on carcinomas of the digestive system (32-37), the pooled sensitivity, specificity, PLR, and NLR of ${ }^{18} \mathrm{~F}-\mathrm{FDG}$ PET/CT were 0.92 (95\% CI, 0.68-0.98), 0.97 (95\% CI, 0.91-0.99), 34.9 (95\% CI, 9.8123.9), and 0.09 (95\% CI, 0.02-0.40), respectively.

\section{Comparison With Conventional Imaging Procedures}

Comparison of the performance of ${ }^{18} \mathrm{~F}$-FDG PET/CT with that of conventional imaging procedures in 7 studies (823 patients) $(1,2,12,29-31,36)$ suggested a major difference in sensitivity (43\%) between ${ }^{18} \mathrm{~F}$-FDG PET/CT and conventional imaging procedures. The pooled sensitivity, specificity, PLR, and NLR of ${ }^{18} \mathrm{~F}-\mathrm{FDG}$ PET/CT were 0.95 (95\% CI, 0.89-0.98), 0.96 (95\% CI, 0.94-0.98), 25.2 (95\% CI, 14.8-43.0), and 0.05 (95\% CI, 0.02-0.12), respectively. The respective values for conventional imaging procedures were 0.52 (95\% CI, 0.31-0.72), 0.89 (95\% CI, 0.80-0.95), 4.9 (95\% CI, 2.9-8.2), and 0.54 (95\% CI, 0.36-0.80).

\section{DISCUSSION}

Early detection of distant malignancies in cancer patients is crucial for guiding subsequent staging procedures and treatment. In this meta-analysis, we included all studies about whole-body PET/CT instead of conventional imaging procedures. Histopathologic analysis or clinical and imaging follow-up was used as the reference standard. We considered $41 \mathrm{PET} / \mathrm{CT}$ studies (4,305 patients) for inclusion in the metaanalysis and quantified the pooled sensitivities and specificities of all 41 PET/CT studies. We found that the sensitivity and specificity of whole-body PET/CT were 0.93 (95\% CI, $0.88-0.96$ ) and 0.96 (95\% CI, 0.95-0.96), respectively. 


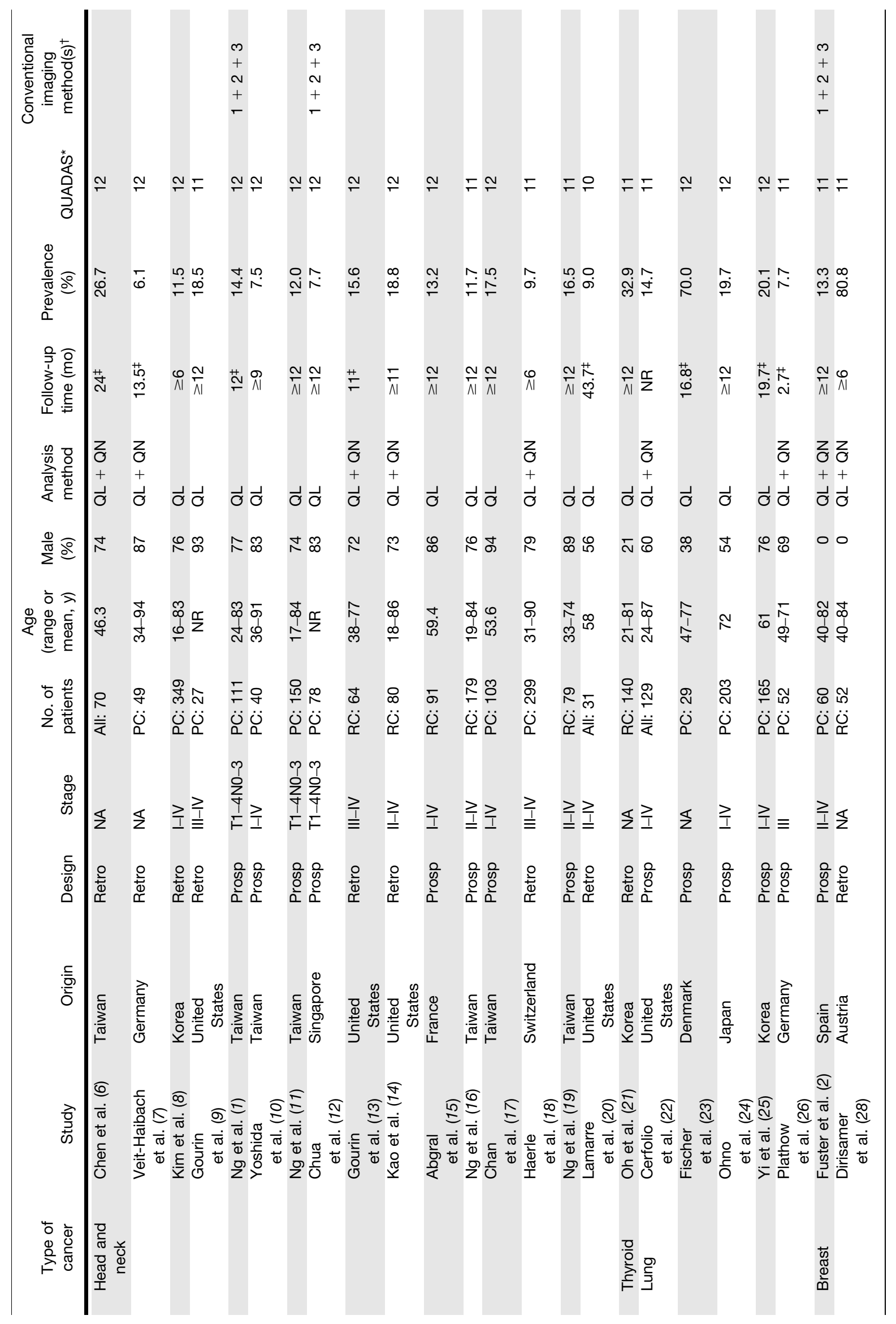

1850 The Journal of Nuclear Medicine • Vol. 53 - No. 12 - December 2012 


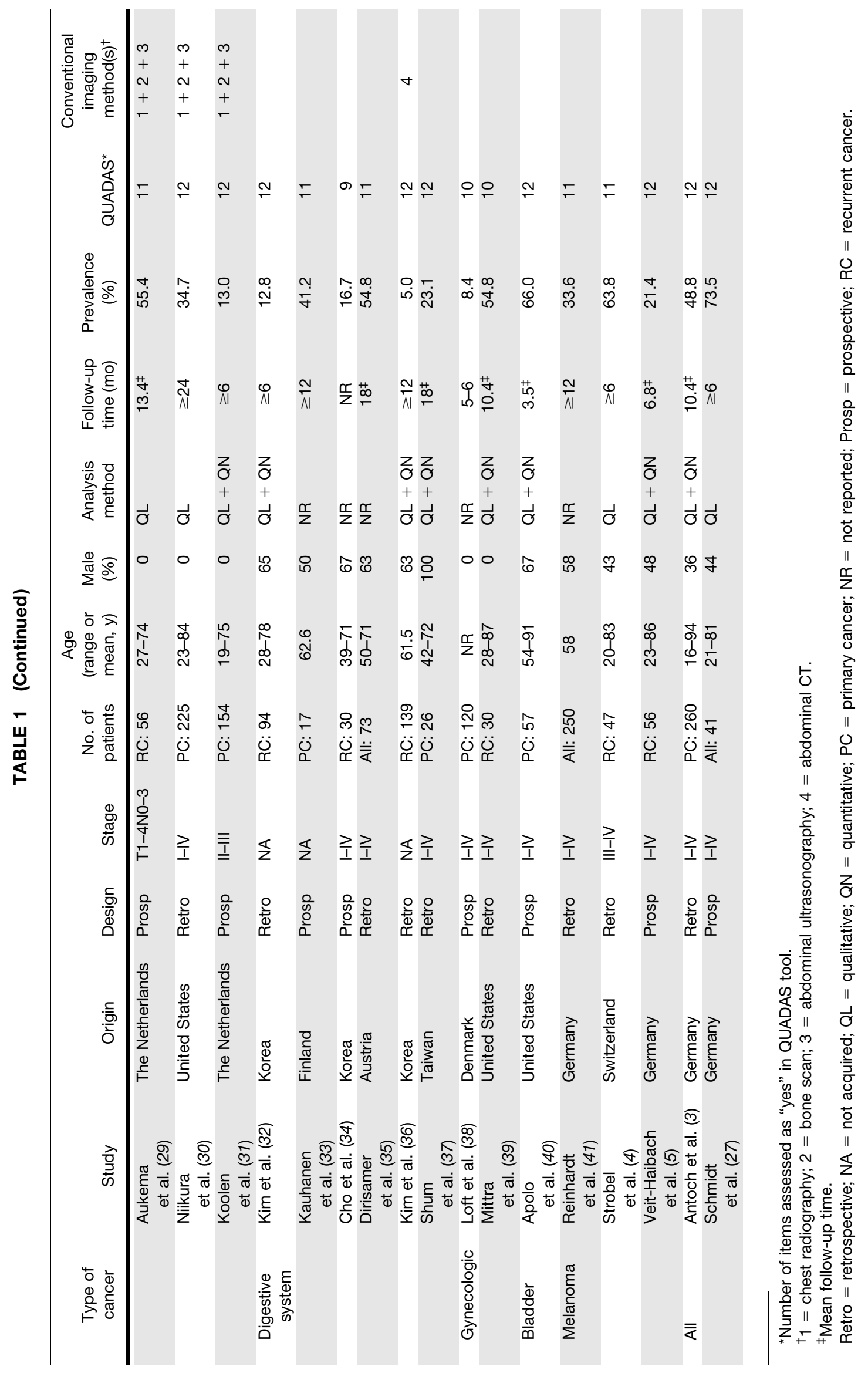


TABLE 2

Diagnostic Accuracy of PET/CT for Overall Evaluation of Distant Malignancies in Cancer Patients

\begin{tabular}{|c|c|c|c|c|c|}
\hline Study & $\begin{array}{l}\text { No. of studies } \\
\text { (no. of patients) }\end{array}$ & Sensitivity $(95 \% \mathrm{Cl})$ & Specificity $(95 \% \mathrm{Cl})$ & PLR (95\% Cl) & NLR $(95 \% \mathrm{Cl})$ \\
\hline All & $41(4,305)$ & $0.93(0.88-0.96)$ & $0.96(0.95-0.96)$ & $20.8(16.8-25.8)$ & $0.08(0.05-0.13)$ \\
\hline \multicolumn{6}{|l|}{ Type of eligible patients } \\
\hline Primary cancer & $23(2,607)$ & $0.91(0.83-0.95)$ & $0.95(0.94-0.96)$ & $18.7(14.9-23.5)$ & $0.10(0.06-0.18)$ \\
\hline Recurrent cancer & $16(1,215)$ & $0.91(0.80-0.96)$ & $0.96(0.94-0.97)$ & $22.9(15.3-34.3)$ & $0.09(0.04-0.22)$ \\
\hline \multicolumn{6}{|l|}{ Prevalence } \\
\hline Low prevalence $(<15 \%)$ & $17(1,825)$ & $0.93(0.85-0.97)$ & $0.96(0.94-0.97)$ & $22.0(16.4-29.7)$ & $0.07(0.03-0.16)$ \\
\hline High prevalence ( $\geq 15 \%)$ & $24(2,480)$ & $0.92(0.84-0.96)$ & 0.95 (0.94-0.97) & 19.7 (14.5-26.9) & $0.09(0.04-0.17)$ \\
\hline \multicolumn{6}{|l|}{ QUADAS* } \\
\hline$<12$ & $18(1,671)$ & $0.95(0.90-0.98)$ & $0.96(0.95-0.97)$ & $25.3(17.3-37.1)$ & $0.05(0.02-0.11)$ \\
\hline$\geq 12$ & $23(2,634)$ & $0.89(0.81-0.94)$ & $0.95(0.94-0.96)$ & $18.1(14.0-23.3)$ & $0.11(0.06-0.21)$ \\
\hline \multicolumn{6}{|l|}{ Analysis method } \\
\hline Qualitative & $19(2,144)$ & $0.90(0.81-0.95)$ & $0.95(0.94-0.96)$ & $18.6(14.8-23.3)$ & $0.11(0.05-0.21)$ \\
\hline Both qualitative and quantitative & $17(1,671)$ & $0.93(0.85-0.97)$ & $0.95(0.93-0.97)$ & $19.7(13.7-28.4)$ & $0.07(0.03-0.17)$ \\
\hline \multicolumn{6}{|l|}{ Design } \\
\hline Prospective & $22(2,071)$ & $0.88(0.79-0.94)$ & $0.96(0.95-0.97)$ & $20.7(16.1-26.7)$ & $0.12(0.07-0.23)$ \\
\hline Retrospective & $19(2,234)$ & $0.96(0.90-0.98)$ & $0.95(0.94-0.97)$ & $20.6(14.5-29.2)$ & $0.04(0.02-0.11)$ \\
\hline
\end{tabular}

*Number of items assessed as "yes" in QUADAS tool.

Across 7 studies (823 patients), whole-body PET/CT had a higher sensitivity ( 0.95 vs. 0.52 ) than conventional imaging procedures. This meta-analysis documented that whole-body PET/CT has excellent diagnostic performance for the overall evaluation of distant metastases with or without second primary cancers in cancer patients.

Because HSROC curves are not easy to interpret and use in clinical practice and because likelihood ratios are considered to be more clinically meaningful, both PLRs

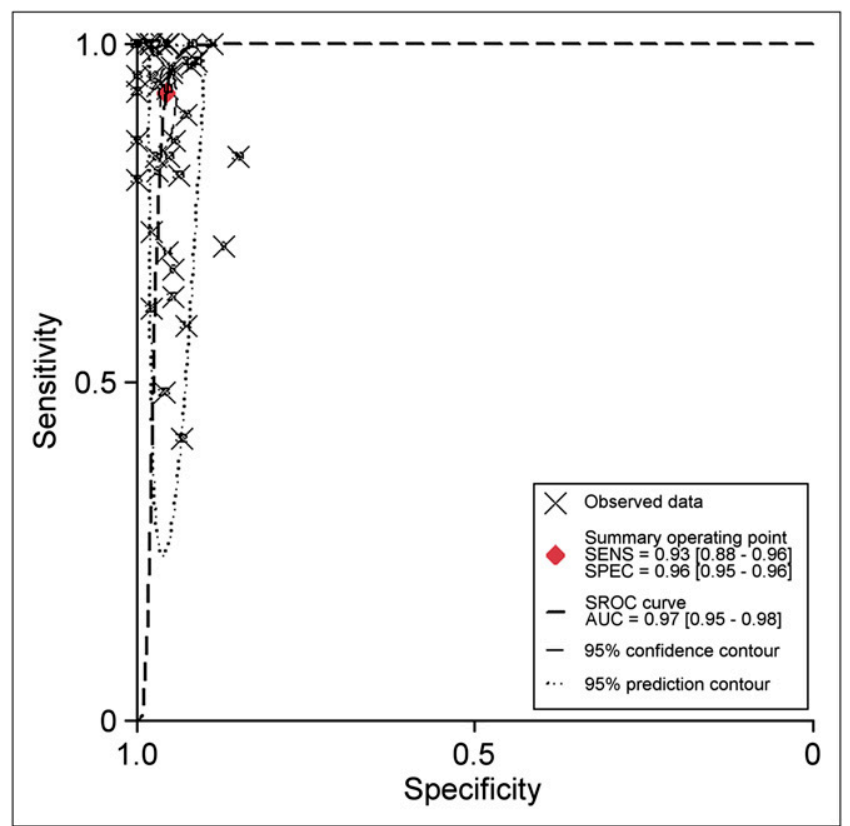

FIGURE 2. HSROC (SROC) curve for diagnostic performance of whole-body PET/CT in all studies. SENS = sensitivity; SPEC = specificity. and NLRs served as our measures of diagnostic accuracy. Discriminating ability is better with higher PLRs and lower NLRs. Although there is no absolute cutoff, a good diagnostic test may have PLRs of greater than 10.0 and NLRs of less than 0.1. The PLR for whole-body PET/CT was 20.7. This value may be high enough to diagnose distant malignancies in cancer patients. On the other hand, the NLR for whole-body PET/CT was 0.08. A negative wholebody PET/CT result may be used alone as a justification to rule out distant malignancies in cancer patients.

The inherent limitations of PET are poor spatial resolution and failure to depict the anatomic structure of disease. Moreover, false-positive findings from inflammatory or granulomatous lesions in regions with a high prevalence of granulomatous disease are still problematic on ${ }^{18} \mathrm{~F}-\mathrm{FDG}$ PET images. These issues may restrict its use for assessing distant malignancies in cancer patients. The poor spatial resolution of PET is substantially compensated for by integrated PET/CT, with coregistration of functional imaging with PET and anatomic imaging with CT. However, little is known about the validity of PET/CT relative to PET for detecting distant malignancies in cancer patients. A retrospective study of 248 patients with various cancers showed that PET/CT had a higher sensitivity than PET alone $(0.94$ vs. $0.78 ; P<0.05)$ and a specificity similar to that of PET alone (0.97 vs. $0.99 ; P>0.05)$ (3). A retrospective study of 250 patients with melanoma also showed that PET/CT had a higher sensitivity than PET alone (0.99 vs. 0.89; $P<$ $0.05)$ and a specificity similar to that of PET alone $(0.98$ vs. $0.95 ; P>0.05)(41)$. Limited prospective evidence supports the notion that integrating PET with CT may obviously improve diagnostic accuracy over that achieved with PET alone. 
This meta-analysis had some limitations. First, there was no standard follow-up strategy or time. This factor may have affected the accuracy of whole-body PET/CT for the detection of distant malignancies in cancer patients. Second, selective reporting bias is a well-known threat for many clinical research fields, including diagnostic tests. The effect, if present, would be in favor of whole-body PET/CT. The exclusion of conference abstracts and letters to the editors may also have led to reporting bias. Third, we did not perform subgroup analyses for every location of the primary tumors because doing so would have required individual patient data and the number of included studies was too limited. Fourth, approximately $46 \%$ of the 41 studies were described as being retrospective. The retrospective nature of studies can be considered a limitation because the possibility that imaging observers might have known the diagnostic outcomes of other imaging modalities before assessing the PET/CT results cannot be excluded. Fifth, the sensitivities in the HSROC curves for the included studies were not uniformly high. Nine $(22 \%)$ of the 41 included studies had sensitivities of less than $75 \%$ $(5,10,11,13,19,21,24,25,32)$. No clear reason for the large difference between these studies and other studies was found.

\section{CONCLUSION}

Whole-body PET/CT has excellent diagnostic performance for the detection of distant malignancies in patients with various cancers, especially head and neck cancer, breast cancer, and lung cancer. Large, multicenter, and prospective studies with strict standardization of PET/CT protocols are now needed to investigate the added value of whole-body PET/CT over conventional imaging procedures and could help in establishing whole-body PET/CT as an accurate tool for the detection of distant malignancies in patients with thyroid cancer, bladder cancer, melanoma, and specific types of digestive system cancers.

\section{ACKNOWLEDGMENT}

No potential conflict of interest relevant to this article was reported.

\section{REFERENCES}

1. Ng SH, Chan SC, Yen TC, et al. Staging of untreated nasopharyngeal carcinoma with PET/CT: comparison with conventional imaging work-up. Eur J Nucl Med Mol Imaging. 2009;36:12-22.

2. Fuster D, Duck J, Paredes P, et al. Preoperative staging of large primary breast cancer with $\left[{ }^{18} \mathrm{~F}\right]$ fluorodeoxyglucose positron emission tomography/computed tomography compared with conventional imaging procedures. J Clin Oncol. 2008;26:4746-4751.

3. Antoch G, Saoudi N, Kuehl H, et al. Whole-body dual-modality fluorine-18-2fluo-2-deoxy-D-glucose positron emission tomography and computed tomography (FDG-PET-CT) for tumor staging in solid tumor: comparison with CT and PET. J Clin Oncol. 2005;22:4357-4368.

4. Strobel K, Skalsky J, Kalff V, et al. Tumour assessment in advanced melanoma: value of FDG PET/CT in patients with elevated serum S-100B. Eur J Nucl Med Mol Imaging. 2007;34:1366-1375.
5. Veit-Haibach P, Vogt FM, Jablonka R, et al. Diagnostic accuracy of contrastenhanced FDG PET/CT in primary staging of cutaneous malignant melanoma. Eur J Nucl Med Mol Imaging. 2009;36:910-918.

6. Chen YK, Su CT, Ding HJ, et al. Clinical usefulness of fused PET/CT compared with PET alone or CT alone in nasopharyngeal carcinoma patients. Anticancer Res. 2006;26:1471-1477.

7. Veit-Haibach P, Luczak C, Wanke I, et al. TNM staging with FDG-PET/CT in patients with primary head and neck cancer. Eur J Nucl Med Mol Imaging. 2007;34:1953-1962.

8. Kim SY, Roh JL, Yeo NK, et al. Combined ${ }^{18} \mathrm{~F}$-fluorodeoxyglucose-positron emission tomography and computed tomography as a primary screening method for detecting second primary cancers and distant metastases in patients with head and neck cancer. Ann Oncol. 2007;18:1698-1703.

9. Gourin CG, Watts TL, Williams HT, Patel VS, Bilodeau PA, Coleman TA. Identification of distant metastases with positron-emission tomography-computed tomography in patients with previously untreated head and neck cancer. Laryngoscope. 2008;118:671-675.

10. Yoshida K, Suzuki A, Nagashima T, et al. Staging primary head and neck cancers with ${ }^{18} \mathrm{~F}$-FDG PET/CT: is intravenous contrast administration really necessary? Eur J Nucl Med Mol Imaging. 2009;36:1417-1424.

11. Ng SH, Chan SC, Yen TC, et al. Pretreatment evaluation of distant-site status in patients with nasopharyngeal carcinoma: accuracy of whole-body MRI at 3-Tesla and FDG-PET-CT. Eur Radiol. 2009;19:2965-2976.

12. Chua ML, Ong SC, Wee JT, et al. Comparison of 4 modalities for distant metastasis staging in endemic nasopharyngeal carcinoma. Head Neck. 2009; 31:346-354.

13. Gourin CG, Watts T, Williams HT, Patel VS, Bilodeau PA, Coleman TA. Identification of distant metastases with PET-CT in patients with suspected recurrent head and neck cancer. Laryngoscope. 2009;119:703-706.

14. Kao J, Vu HL, Genden EM, et al. The diagnostic and prognostic utility of positron emission tomography/computed tomography-based follow-up after radiotherapy for head and neck cancer. Cancer. 2009;115:4586-4594.

15. Abgral R, Querellow S, Potard G, et al. Does ${ }^{18}$ F-FDG PET/CT improve the detection of posttreatment recurrence of head and neck squamous cell carcinoma in patients negative for disease on clinical follow-up? J Nucl Med. 2009;50:24-29.

16. Ng SH, Chan SC, Yen TC, et al. Comprehensive imaging of residual/recurrent nasopharyngeal carcinoma using whole-body MRI at $3 \mathrm{~T}$ compared with FDGPET-CT. Eur Radiol. 2010;20:2229-2240.

17. Chan SC, Wang HM, Yen TC, et al. ${ }^{18} \mathrm{~F}-\mathrm{FDG}$ PET/CT and 3.0-T whole-body MRI for the detection of distant metastases and second primary tumours in patients with untreated oropharyngeal/hypopharyngeal carcinoma: a comparative study. Eur J Nucl Med Mol Imaging. 2011;38:1607-1619.

18. Haerle SK, Schmid DT, Ahmad N, Hany TF, Stoeckli SJ. The value of ${ }^{18}$ F-FDG PET/CT for the detection of distant metastases in high-risk patients with head and neck squamous cell carcinoma. Oral Oncol. 2011;47:653-659.

19. Ng SH, Chan SC, Yen TC, et al. PET/CT and 3-T whole-body MRI in the detection of malignancy in treated oropharyngeal and hypopharyngeal carcinoma. Eur J Nucl Med Mol Imaging. 2011;38:996-1008.

20. Lamarre ED, Batra PS, Lorenz RR, et al. Role of positron emission tomography in management of sinonasal neoplasms: a single institution's experience. Am J Otolaryngol. 2012;33:289-295.

21. Oh JR, Byun BH, Hong SP, et al. Comparison of ${ }^{131} \mathrm{I}$ whole-body imaging, ${ }^{131} \mathrm{I}$ SPECT/CT, and ${ }^{18}$ FDG PET/CT in the detection of metastatic thyroid cancer. Eur J Nucl Med Mol Imaging. 2011;38:1459-1468.

22. Cerfolio RJ, Ojha B, Bryant AS, et al. The accuracy of integrated PET-CT compared with dedicated PET alone for the staging of patients with nonsmall cell lung cancer. Ann Thorac Surg. 2004;78:1017-1023.

23. Fischer BM, Mortensen J, Langer SW, et al. A prospective study of PET/CT in initial staging of small-cell lung cancer: comparison with CT, bone scintigraphy and bone marrow analysis. Ann Oncol. 2007;18:338-345.

24. Ohno Y, Koyama H, Onishi Y, et al. Non-small cell lung cancer: whole-body MR examination for M-stage assessment-utility for whole-body diffusionweighted imaging compared with integrated FDG PET/CT. Radiology. 2008; 248:643-654.

25. Yi CA, Shin KM, Lee KS, et al. Non-small cell lung cancer staging: efficacy comparison of integrated PET/CT versus 3.0-T whole-body MR imaging. Radiology. 2008;248:632-642.

26. Plathow C, Aschoff P, Lichy MP, et al. Positron emission tomography/computed tomography and whole-body magnetic resonance imaging in staging of advanced non small cell lung cancer: initial results. Invest Radiol. 2008;43:290-297.

27. Schmidt GP, Baur-Melnyk A, Herzog P, et al. High-resolution whole-body magnetic resonance image tumor staging with the use of parallel imaging versus dual-modality positron emission tomography-computed tomography: experience on a 32-channel system. Invest Radiol. 2005;40:743-753. 
28. Dirisamer A, Halpern BS, Flory D, et al. Integrated contrast-enhanced diagnostic whole-body PET/CT as a first-line restaging modality in patients with suspected metastatic recurrence of breast cancer. Eur J Radiol. 2010;73:294-299.

29. Aukema TS, Rutgers EJ, Vogel WV, et al. The role of FDG PET/CT in patients with locoregional breast cancer recurrence: a comparison to conventional imaging techniques. Eur J Surg Oncol. 2010;36:387-392.

30. Niikura N, Costelloe CM, Madewell JE, et al. FDG-PET/CT compared with conventional imaging in the detection of distant metastases of primary breast cancer. Oncologist. 2011;16:1111-1119.

31. Koolen BB, Vrancken Peeters MJ, Aukema TS, et al. ${ }^{18}$ F-FDG PET/CT as a staging procedure in primary stage II and III breast cancer: comparison withconventional imaging techniques. Breast Cancer Res Treat. 2012;131:117-126.

32. Kim JY, Kim MH, Lee TY, et al. Clinical role of ${ }^{18}$ F-FDG PET-CT in suspected and potentially operable cholangiocarcinoma: a prospective study compared with conventional imaging. Am J Gastroenterol. 2008;103:1145-1151.

33. Kauhanen SP, Komar G, Seppanen MP, et al. A prospective diagnostic accuracy study of ${ }^{18} \mathrm{~F}$-fluorodeoxyglucose positron emission tomography/computed tomography, multidetector row computed tomography, and magnetic resonance imaging in primary diagnosis and staging of pancreatic cancer. Ann Surg. 2009;250:957-963.

34. Cho YB, Chun HK, Kim HJ, et al. Accuracy of MRI and ${ }^{18} \mathrm{~F}-\mathrm{FDG}$ PET/CT for restaging after preoperative concurrent chemoradiotherapy for rectal cancer. World J Surg. 2009;33:2688-2694.
35. Dirisamer A, Halpern BS, Flory D, et al. Performance of integrated FDG-PET/ contrast-enhanced CT in the staging and restaging of colorectal cancer: comparison with PET and enhanced CT. Eur J Radiol. 2010;73:324-328.

36. Kim DW, Park SA, Kim CG. Detecting the recurrence of gastric cancer after curative resection: comparison of FDG PET/CT and contrast-enhanced abdominal CT. J Korean Med Sci. 2011;26:875-880.

37. Shum WY, Hsieh TC, Yeh JJ, et al. Clinical usefulness of dual-time FDG PETCT in assessment of esophageal squamous cell carcinoma. Eur J Radiol. 2012; 81:1024-1028.

38. Loft A, Berthelsen AK, Roed H, et al. The diagnostic value of PET/CT scanning in patients with cervical cancer: a prospective study. Gynecol Oncol. 2007;106: 29-34.

39. Mittra E, El-Maghraby M, Cesar A, et al. Efficacy of ${ }^{18}$ F-FDG PET/CT in the evaluation of patients with recurrent cervical carcinoma. Eur J Nucl Med Mol Imaging. 2009;36:1952-1959.

40. Apolo AB, Riches J, Schoder H, et al. Clinical value of fluorine-18-2-fluo-2deoxy-D-glucose positron emission tomography/computed tomography in bladder cancer. J Clin Oncol. 2010;28:3973-3978.

41. Reinhardt MJ, Joe AY, Jaeger U, et al. Diagnostic of performance of whole body dual modality ${ }^{18} \mathrm{~F}$-FDG PET/CT imaging for $\mathrm{N}$ - and M-staging of malignant melanoma: experience with 250 consecutive patients. J Clin Oncol. 2006;24: 1178-1187. 